\title{
OPTICAL DIAGNOSTIC AND MODELING SOLUTION GROWTH PROCESS OF SODIUM CHLORATE CRYSTALS *
}

\author{
WANG Tao (王涛)， DUAN Li (段俐) \\ (National Microgravity Laboratory, Institute of Mechanics, Chinese Academy of Sciences, \\ Beijing 100080, P. R. China) \\ (Communicated by HU Wen-rui)
}

\begin{abstract}
Both a real time optical interferometric experiment and a numerical simulation of two-dimension non-steady state model were employed to study the growth process of aqueous sodium chlorate crystals. The parameters such as solution concentration distribution, crystal dimensions, growth rate and velocity field were obtained by both experiment and numerical simulation. The influence of earth gravity during crystal growth process was analyzed. A reasonable theory model corresponding to the present experiment is advanced. The thickness of concentration boundary layer was investigated especially. The results from the experiment and numerical simulation match well.
\end{abstract}

Key words: crystal growth; interferometry; numerical simulation; concentration boundary layer

Chinese Library Classification: O781

2000 Mathematics Subject Classification: 74N05; 74N25

Digital Object Identifier(DOI): 10.1007/s 10483-006-0904-1

\section{Introduction}

Crystal growth is a procedure of phase changing. It is influenced by kinds of physical phenomena. Since structure details of crystal growth process from either melt or solution are still not fully understood, a complete convincing theory model is not advanced by now. The quality and shape of crystals in growth process are known to be affected by many factors, such as impurities, steps bunching, and the transport process of diffusion and convection. Further more there is a little difference in microgravity condition between that in terrestrial gravity. So, more and more researches in this field are carried out to discover the physical essentials and to improve the growth model.

According to the larger crystal scale, ground-based inorganic crystal growth experiment is much more facile for optical observing than that of protein crystal. And the experiments have obtained many significant results. Onuma and Tsukamoto developed the phase shift optic interface method with high resolution ${ }^{[1,2]}$, and Duan et al. improved it to show the concentration transfer ${ }^{[3-5]}$. This Mach-Zehnder interferometry system can be used for real time observation of concentration field and velocity field. Then experiment data are processed by an image process system to obtain the crystal growth rate and the thickness of concentration boundary layer. In this paper, a same Mach-Zehnder interferometry system is employed to observe the growth process of $\mathrm{NaClO}_{3}$ crystals.

Modeling the process of crystal growth is an attractive problem interests not only dynamicists but also geologists, biologist (especially in biostructure) and fluid physicists ${ }^{[6-10]}$. Many

* Received Nov.1, 2005; Revised Jun.1, 2006

Project supported by the National Natural Science Foundation of China (No.10432060)

Corresponding author DUAN Li, Associate Professor, Doctor, E-mail: duanli@imech.ac.cn 
growth procedures in different environments including melt growth, solution growth and vapor diffusion are widely discussed recently ${ }^{[7-9,11]}$. In present paper, a two-dimension linear convection-diffusion model is advanced to match the aqueous $\mathrm{NaClO}_{3}$ crystallization experiment. The crystallization experiment and the numerical simulation are both discussed in present paper to obtain the mechanical essentials of the growth process under earth gravity. According to the experiment results, the reasonability of the growth model is validated.

\section{Theoretical Analysis and Model}

The terrestrial crystal growth process is inevitably affected by earth gravity. The gradient of solution concentration brings on layer deposition, which turns into buoyancy convection under earth gravity. As the crystal growth process is affected by the buoyancy convection, it may cause crystal defects in dimension, integrality, optical uniformity, etc. The process of crystal growth is considered as a combination of the bulk transport and the interface kinetics ${ }^{[0,10,12]}$. In the bulk transport procedure solute is transported to the growth interface from the bulk, while in the interface kinetics procedure the solute on the growth interface moves to the kinks to enter the crystal lattice. Bulk transport is controlled by the difference of bulk concentration $C$ and surface concentration $C_{\mathrm{s}}$, and interface kinetics is driven by the difference of surface concentration $C_{\mathrm{s}}$ and equilibrium concentration $C_{\text {eq }}$. The two procedures couple to conduct the whole growth process. In extreme situation of absolute bulk transport surface concentration approach to equilibrium concentration: $C_{\mathrm{s}} \rightarrow C_{\mathrm{eq}}$, while in the opposite absolute interface kinetics situation $C_{\mathrm{s}} \rightarrow C^{[6,8,10]}$. A simplified two-dimensional model is employed to simulate the non-steady process of the terrestrial crystal growth in Boussinesq approximation. A coordinate system which fixed on the upper crystal surface is considered. The process is governed by the following equations $^{[6,7,10]}$ :

$$
\begin{aligned}
& \frac{\partial u}{\partial x}+\frac{\partial v}{\partial y}=0 \\
& \frac{\partial u}{\partial t}+u \frac{\partial u}{\partial x}+v \frac{\partial u}{\partial y}=-\frac{\partial P}{\partial x}+\nu\left(\frac{\partial^{2} u}{\partial x^{2}}+\frac{\partial^{2} u}{\partial y^{2}}\right) \\
& \frac{\partial v}{\partial t}+u \frac{\partial v}{\partial x}+v \frac{\partial v}{\partial y}=-\frac{\partial P}{\partial y}+\nu\left(\frac{\partial^{2} v}{\partial x^{2}}+\frac{\partial^{2} v}{\partial y^{2}}\right)-g \beta_{c}\left(C-C_{0}\right) \\
& \frac{\partial C}{\partial t}+u \frac{\partial C}{\partial x}+v \frac{\partial C}{\partial y}=D\left(\frac{\partial^{2} C}{\partial x^{2}}+\frac{\partial^{2} C}{\partial y^{2}}\right)
\end{aligned}
$$

Boundary conditions at the crystal growth cell wall:

$$
\begin{aligned}
& \frac{\partial C}{\partial n}=0, \\
& \left.u\right|_{x=-a, a}=0,\left.\quad v\right|_{x=-a, a}=0 .
\end{aligned}
$$

Boundary conditions at the solution-atmosphere interface (free interface):

$$
\begin{aligned}
& P=P_{0}, \\
& C=C_{\text {eva }}, \\
& \left.v\right|_{y=l}=0 .
\end{aligned}
$$


Boundary condition at the growth interface (Fick's law):

$$
D \frac{\partial C}{\partial x}=K\left(C-C_{\mathrm{eq}}\right)
$$

Initial condition:

$$
C=C_{0}
$$

Here $u, v$ are the solution velocity of $x$-, $y$-direction, respectively, $\nu$ is the kinematic viscosity, $\beta_{c}$ is the coefficient of solutal expansion, $D$ is the solute diffusivity, $n$ is the normal direction of the cell wall, $P_{0}$ is the atmospheric pressure, $C_{\text {eva }}$ is the steady evaporation concentration of the free surface, and $C_{0}$ is the initial solution concentration.

Commercially available code FLUENT is used for numerical simulation. A $96 \times 96$ mesh is used to correspond an area of $12 \mathrm{~mm} \times 12 \mathrm{~mm}$ of actual dimension. The 4 rows mesh near the crystal are fined (Fig.(1)). The finer mesh shows a much clearer result of velocity field and concentration distribution near the interface. At the side-wall and the bottom of the growth cell, it is given a non-diffusive flux boundary condition (Eq.(5)). The upper surface, which is a free evaporation surface, is given a fixed evaporation surface concentration (Eq.(8)) as the environment parameters keep invariable such as temperature and humidity. The seed crystal of $3 \mathrm{~mm} \times 1 \mathrm{~mm}$ dimension is placed on the bottom center of the growth cell. Boundary condition at the growing interface is given by the Fick's law (Eq.(10)). This model is a simulation of the actual experiment with similar geometry scales and the same boundary and initial conditions. So the results obtained from the numerical simulation are comparable to those of the actual experiment. Thus the physical process of growth can be properly investigated.

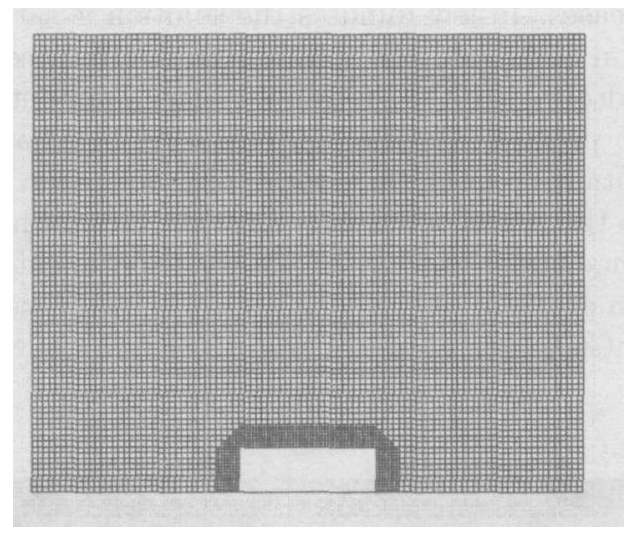

Fig.1 Mesh used in simulation

\section{Experiment of Crystal Growth and Results}

A set of Mach-Zehnder interferometer system (Fig.(2)) is employed in the experiment to obtain the concentration gradient of the solution fluid field in the growth process. In the MachZehnder interferometer system, a beam of laser passes through a group of lens (a) to form an expended parallel light beam, and then the beam is split by a splitter (b) into two beams. One beam of laser, which crosses the crystal growth cell (c), is used as the object beam, and the other is used as the reference beam. The two beams meet at a certain point and form an interferometric fringe pattern, which carries out the concentration gradient. Then fringe images are received by a CCD camera (f) and recorded by computer with a Matrox image capture card. And later these images are processed by computer. 


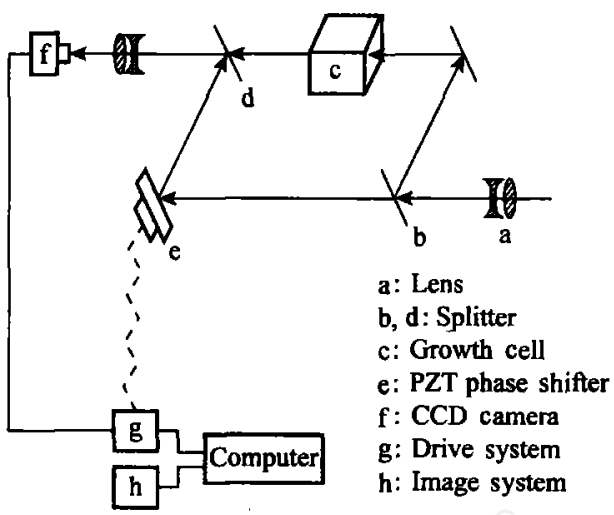

Fig.2 Schematics of experiment set

The aqueous growth of sodium chlorate crystals is studied in present experiment. The $48.0 \%$ concentration of $\mathrm{NaClO}_{3}$ solution prepared at $18^{\circ} \mathrm{C}$ is injected into the crystal growth cell of $12 \mathrm{~mm} \times 9 \mathrm{~mm} \times 10 \mathrm{~mm}$ dimension, which is made of optical glass. A seed crystal of $2.78 \mathrm{~mm} \times 1.32 \mathrm{~mm} \times 0.72 \mathrm{~mm}$ dimension is placed on the bottom of growth cell. The upper surface of the crystal is face $\left(\begin{array}{lll}0 & 0 & 1\end{array}\right)$. The left and right faces are faces $\left(\begin{array}{lll}0 & 1 & 0\end{array}\right)$. Environment temperature is kept at $18^{\circ} \mathrm{C}$ invariably during the whole process of experiment. At the very beginning, the solution is under saturated, but due to the dissolution and evaporation, the solution concentration increases. In few minutes the solution is saturated, and the dissolution stopped. As the evaporation continues, the solution gradually becomes supersaturated. Thus solute near the crystal is adsorbed to the growth interface, and enters the crystal lattice after a complex interface kinetic process. As the concentration in adjacent volume decreases, the density of this part of solution is lower than the bulk. So under the earth gravity, a plumb flow from crystal surface to the top of solution is formed. This is the buoyancy convection as a result of concentration change under gravity. In present experiment, the interferometric fringes related to the concentration gradient of the solution are shown clearly in the captured images. After 6 hours' growth, a $\mathrm{NaClO}_{3}$ crystal of $4.56 \mathrm{~mm} \times 2.30 \mathrm{~mm} \times 1.18 \mathrm{~mm}$ dimension is obtained (Fig.(3)).

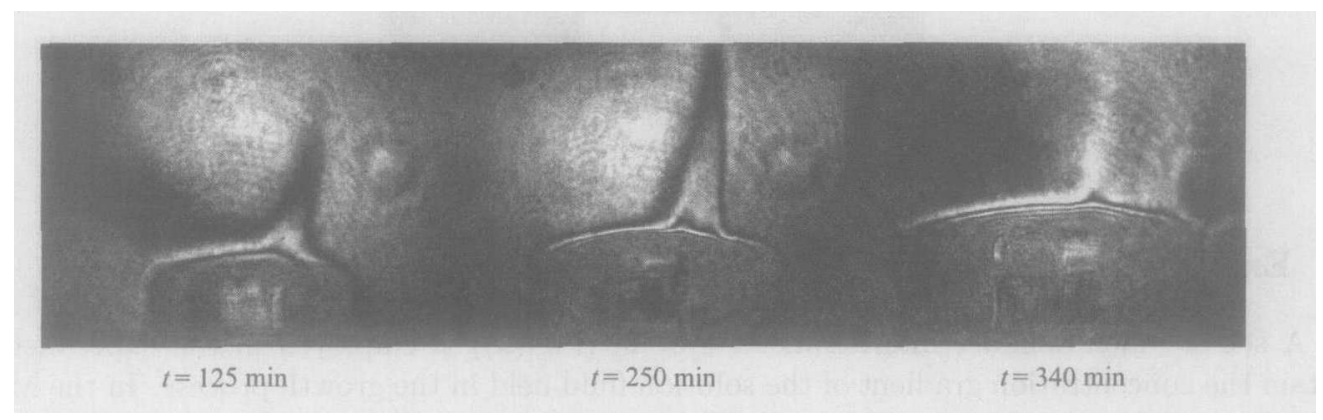

Fig.3 Evolution of interferometric fringe

Interferometric fringes from experiment image data carry out the concentration distribution of the fluid field. As shown in Fig.(4), a four-step phase shift image Fig.4(a) is interpreted in phase calculation and phase unwrapping to obtain the solution concentration distribution (Fig.4(b)) at a certain time. In Fig.(4), the experiment time is $t=240 \mathrm{~min}$. 
The concentration distribution obtained from phase calculation and phase unwrapping also shows the diffusion boundary layer. These images demonstrate that the concentration near the growth interface decreases rapidly to form a growth boundary layer. As shown in Fig.4(c), according to the concentration curve at $Z=3.15 \mathrm{~mm}$, the thickness of the concentration boundary layer is approximately $200 \mu \mathrm{m}$.

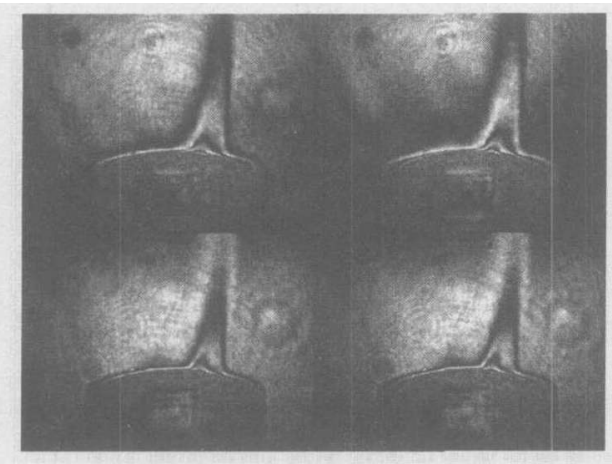

(a) Four-step phase shift interferometric fringes in crystal growth process

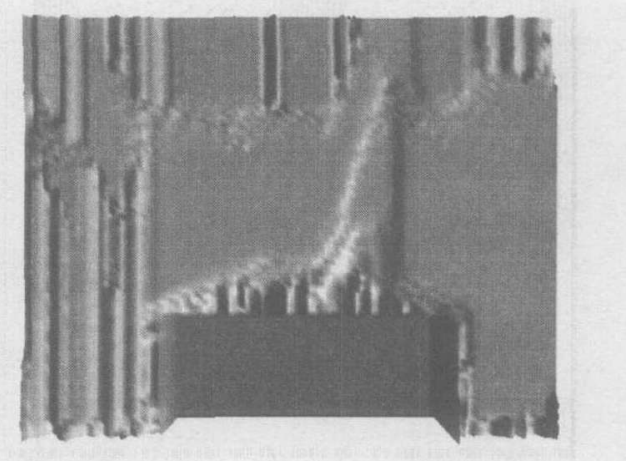

(b) Concentration distribution



(c) Concentration distribution curve at horizontal line $Z=3.15 \mathrm{~mm}$

Fig.4 Concentration distribution at $t=240 \mathrm{~min}$

\section{Numerical Simulation}

Numerical simulation of the non-steady model introduced in section 1 is used to calculate the fluid field of crystal growth process. The solution parameters at $t=240 \mathrm{~min}$ are taken as a sample. The velocity field is shown in Figs.5(a) and 5(b), and the concentration distribution in Figs.5(c) and 5(d). As a result of the earth gravity, plumb flow of buoyancy convection is formed in the fluid field Fig.5(a). At the area closed to face $\left(\begin{array}{lll}0 & 0 & 1\end{array}\right)$, flow moves vertically to the upper evaporation surface. The flow near the crystal is faster than the other part of the flow field. The crystal is enwrapped by the flow near the interface Fig.5(b).

Concentration near the growth interface reduces rapidly to the surface concentration $C_{\mathrm{s}}$. Thus a concentration boundary layer is formed. Meantime at the upper surface of the growth cell, the concentration changes rapidly from the invariable evaporation concentration to the 




(a) Velocity field

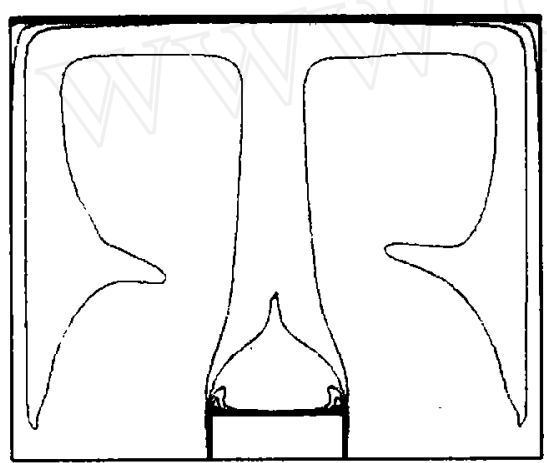

(c) Concentration distribution



(b) Velocity field near growth interface .

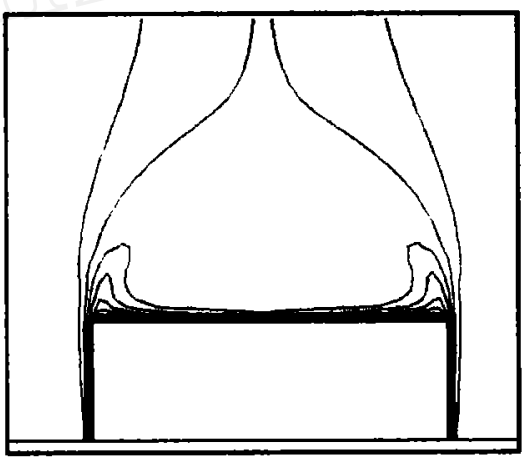

(d) Concentration distribution near growth interface

Fig.5 Velocity field and concentration distribution at $t=240 \mathrm{~min}$

solution concentration as well. In the other part of the solution, the concentration remains at a certain level, although the buoyancy convection changes the concentration distribution.

Velocity field and the concentration distribution are calculated by the same simulation model at $t=15 \mathrm{~min}$ and $t=50 \mathrm{~min}$ (Fig.6). And the results of calculation is synthetically analyzed to study the evolvement of velocity field and concentration field in the entire process of crystal growth together with those at $t=240 \mathrm{~min}$. It is demonstrated that the solute in the adjacent volume of the growth interface is firstly adsorbed at the very beginning, and a plumb convection is formed as the density reduced gradually. After a certain period of growth, the velocity distribution and the concentration distribution are prone to be stabile until the end of the growth process.

Considering the calculation of concentration distribution (Figs.5(c),5(d),6(c),6(d)), the boundary layer near face $\left(\begin{array}{lll}0 & 1 & 0\end{array}\right)$ changes slightly due to the influence of the convection. And the thickness remains at a certain value. At the time $t=240 \mathrm{~min}$, the thickness of the boundary layer is $166 \mu \mathrm{m}$, which approximately matches that of experiment data $200 \mu \mathrm{m}$ (Fig.(7)). All the few main steps of the growth process occur in the concentration boundary layer, including the transportation of solute from solution to the interface and the entrance into crystal lattices. Thus the research of concentration boundary layer is of practical significance. In following experiments, equipments with even higher resolution are required to obtain more effective results. 


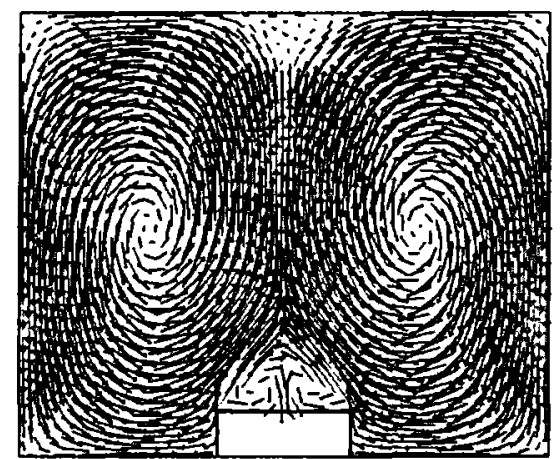

(a) Velocity field at $t=15 \mathrm{~min}$

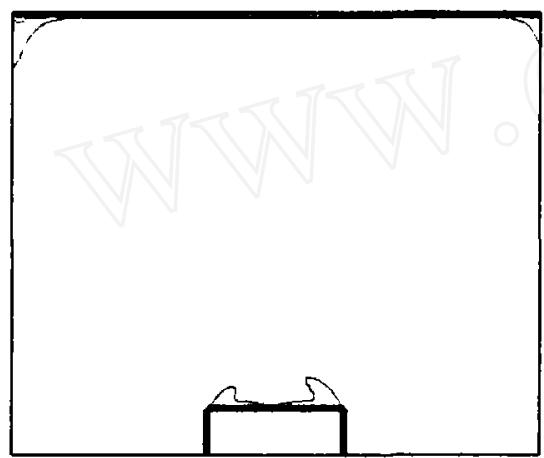

(c) Concentration distribution at $t=15 \mathrm{~min}$

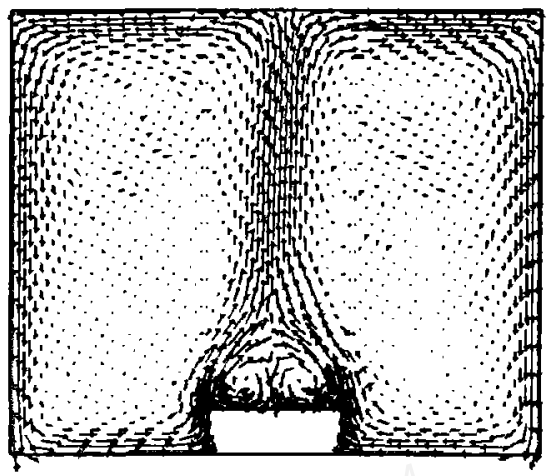

(b) Velocity field at $t=50 \mathrm{~min}$

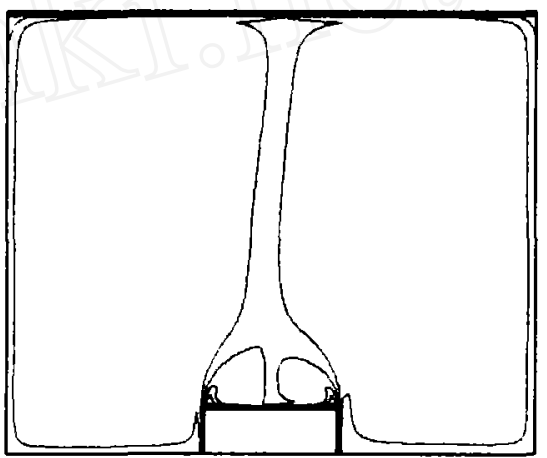

(d) Concentration distribution at $t=50 \mathrm{~min}$

Fig.6 Evolution of velocity field and concentration distribution



Fig.7 Concentration distribution curve of simulation results and experiment data at the horizontal line $Z=3.15 \mathrm{~mm}, t=240 \mathrm{~min}$ ( $X=0$ is the growth interface)

\section{Conclusions}

The experiment data provide evolvement of several physical parameters during the growth process such as the crystal dimension, concentration distribution, thickness of the concentration boundary layer, etc. And the numerical simulation of the two-dimension non-steady model 
gives the same parameters by calculation. Comparing the two methods, the simulation results mostly match those from experiment. Thus this model does correspond to the actual situation of present experiment, especially concerning the parameters of the solution fluid field. As in the inorganic crystal growth process, the influence of nonlinear process is not quite obvious because of large crystal scale and high growth rate, the nonlinear interface kinetic process is not discussed in this model. In further discussions of protein crystal growth process, when the influence could not be neglected, the nonlinear kinetics may play a more important role to the numerical simulation. To advance a proper assumption to the interface nonlinear kinetics of protein crystal growth process is the main objective of further researches.

\section{References}

[1] Onuma K, Tsukamoto K, Sunagawa I. Role of buoyancy driven convection in aqueous solution growth: a case study of $\left(\mathrm{BaNO}_{3}\right)_{2}$ crystal[J]. J Crystal Growth, 1988, 89(2-3):177-188.

[2] Onuma K, Kameyama T, Tsukamoto K. In situ study of surface phenomena by real time phase shift interferometry[J]. J Crystal Growth, 1994, 137(3-4):610-622.

[3] Duan L, Kang Q, Hu W R. The mass transfer process and the growth rate of protein crystals[J]. Biophysical Chemistry, 2002, 97(2-3):189-201.

[4] Duan L, Shu J Z. The convection during $\mathrm{NaClO}_{3}$ crystal growth observed by the phase shift interferometer[J]. J. Crystal Growth, 2001, 223(1-2):181-188.

[5] Duan L, Kang Q, Shu J Z. Experimental study on the mini-crystal growth by optical diagnostics[J]. Acta Mechanica Sinica, 2002, 34(3):463-468 (in Chinese).

[6] Hu W R. Xu S C. Fluid Mechanics in Microgravity[M]. Science Press, Beijing, 1999, 182-192 (in Chinese).

[7] Nadarjah Arunan, Rosenberger Franz, Alexander J Iwan D. Modelling the solution growth of TGS crystals in low gravity[J]. J Crystal Growth, 1990, 104(2):218-232.

[8] Vekilov Peter G, Alexander J Iwan D, Rosenberger Franz. Nonlinear response of layer growth dynamics in the mixed kinetics-bulk-transport regime[J]. Physical Review E, 1996, 54(6):66506660.

[9] Vekilov Peter G, Lin Hong, Rosenberger Franz. Unsteady crystal growth due to step-bunch cascading[J]. Physical Review E, 1997, 55(3):3202-3214.

[10] Allégre Claude J, Provost Ariel, Jaupart Claude. Oscillatory zoning: a pathological case of crystal growth[J]. Nature, 1981, 294(5838):223-228.

[11] Kang Q, Duan L, Hu W R. Mass transfer process during the $\mathrm{NaClO}_{3}$ crystal growth process[J]. Int J Heat Mass Transfer, 2001, 44(17):3213-3222.

[12] Zhang K C, Zhang Y D. Science and Technology in Crystal Growth[M]. 2nd Edition. Science Press, Beijing, 1997, 107-124 (in Chinese). 